\title{
Volatile profiling reveals intracellular metabolic changes in Aspergillus parasiticus: veA regulates branched chain amino acid and ethanol metabolism
}

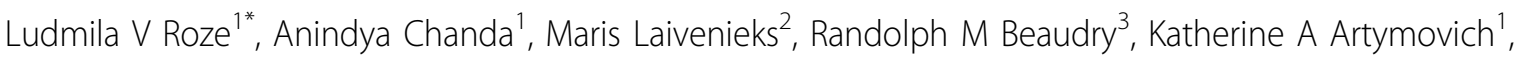
Anna V Koptina', Deena W Awad', Dina Valeeva', Arthur D Jones ${ }^{4,5}$, John E Linz ${ }^{1,2,6}$

\begin{abstract}
Background: Filamentous fungi in the genus Aspergillus produce a variety of natural products, including aflatoxin, the most potent naturally occurring carcinogen known. Aflatoxin biosynthesis, one of the most highly characterized secondary metabolic pathways, offers a model system to study secondary metabolism in eukaryotes. To control or customize biosynthesis of natural products we must understand how secondary metabolism integrates into the overall cellular metabolic network. By applying a metabolomics approach we analyzed volatile compounds synthesized by Aspergillus parasiticus in an attempt to define the association of secondary metabolism with other metabolic and cellular processes.
\end{abstract}

Results: Volatile compounds were examined using solid phase microextraction - gas chromatography/mass spectrometry. In the wild type strain Aspergillus parasiticus SU-1, the largest group of volatiles included compounds derived from catabolism of branched chain amino acids (leucine, isoleucine, and valine); we also identified alcohols, esters, aldehydes, and lipid-derived volatiles. The number and quantity of the volatiles produced depended on media composition, time of incubation, and light-dark status. A block in aflatoxin biosynthesis or disruption of the global regulator veA affected the volatile profile. In addition to its multiple functions in secondary metabolism and development, VeA negatively regulated catabolism of branched chain amino acids and synthesis of ethanol at the transcriptional level thus playing a role in controlling carbon flow within the cell. Finally, we demonstrated that volatiles generated by a veA disruption mutant are part of the complex regulatory machinery that mediates the effects of VeA on asexual conidiation and sclerotia formation.

Conclusions: 1) Volatile profiling provides a rapid, effective, and powerful approach to identify changes in intracellular metabolic networks in filamentous fungi. 2) VeA coordinates the biosynthesis of secondary metabolites with catabolism of branched chain amino acids, alcohol biosynthesis, and $\beta$-oxidation of fatty acids. 3) Intracellular chemical development in A. parasiticus is linked to morphological development. 4) Understanding carbon flow through secondary metabolic pathways and catabolism of branched chain amino acids is essential for controlling and customizing production of natural products.

\footnotetext{
* Correspondence: roze@msu.edu

'Department of Food Science and Human Nutrition, Michigan State

University, East Lansing, MI, USA

Full list of author information is available at the end of the article
}

(C) 2010 Roze et al; licensee BioMed Central Ltd. This is an Open Access article distributed under the terms of the Creative Commons 


\section{Background}

Secondary metabolites are low-molecular-weight natural products generated by filamentous fungi, plants, algae, bacteria, and animals in response to environmental abiotic and biotic stimuli. Secondary metabolites have a strong impact on humankind via their application in health, medicine, agriculture, and industry; they include useful (e.g. antibiotics) and detrimental compounds (e.g. mycotoxins).

Filamentous fungi produce a broad range of secondary metabolites. Each fungal species can synthesize multiple secondary metabolites, and these metabolites vary from species to species as well thus enabling the use of secondary metabolite profiling in the chemotaxonomy of filamentous fungi [1-3]. The complex network of secondary metabolism is connected to basic (primary) metabolism. Secondary metabolites are derived from compounds formed during primary metabolism, e.g. amino acids, nucleotides, carbohydrates, acyl-CoA (reviewed in [4]). Each secondary metabolic pathway accomplishes its specific function (although often unknown) as part of cellular metabolism and appears to provide "active safety" mechanisms for the producer enhancing survival in the continuously changing environment [5-10].

Aspergillus spp. produce an array of secondary metabolites including aflatoxin, cyclopiazonic acid, aflatrem, patulin, penicillin, kojic acid, lovastatin, carotenoids, and spore pigments; novel secondary metabolites have also been discovered that are synthesized from so called silent gene clusters in $A$. nidulans, such as terrequinone A, monodictyphenone, emodins, and polyketides [11]. Fungal-bacterial physical interactions have been shown to induce silent secondary metabolic gene cluster expression in A. nidulans required for biosynthesis of the polyketide orsellinic acid $[5,9]$.

Aflatoxin biosynthesis is one of the most highly characterized secondary metabolic pathways [12-15]. In contrast, pathways for the synthesis of many other secondary metabolites, e.g. patulin, cyclopiazonic acid, aflatrem, and kojic acid, are poorly understood [16-18]. Molecular regulation of aflatoxin biosynthesis is complex and involves control of gene expression at the level of the individual gene and at the level of the entire gene cluster $[14,15,19]$. Biosynthesis of aflatoxin initiates during a transition from exponential growth to stationary phase, and closely correlates with fungal development (conidiospore, cleistothecia, and sclerotia formation) [20-22]. AflR, a positive aflatoxin pathway regulator, is a transcription factor that controls at least in part expression of several genes in the aflatoxin gene cluster [23]. VeA, a global regulator of secondary metabolism, links response to light with secondary metabolism and fungal development; this response is mediated through formation of a protein complex VelB/VeA/LaeA [24]. LaeA is a nuclear methyl transferase that through protein-protein interactions mediates regulation of secondary metabolism and development [21]. Aflatoxin biosynthesis is precisely orchestrated within the cell; the early reactions are reported to occur in peroxisomes [25]; recent evidence from our laboratory suggests that specific early steps as well the middle and late steps are carried out in specialized trafficking vesicles, called aflatoxisomes, which are also involved in export of the toxin outside the cell [12]. A novel role for $\mathrm{VeA}$ in coordination of aflatoxisome development with aflatoxin biosynthesis was recently discovered [4,12]. Biosynthesis of aflatoxins appears to fulfill multiple biochemical and biological functions including removal of acetate, protection of the genome from UV damage [26], quenching oxidative stress [27-29], protection from insects [30,31], and regulation of conidiation, and sclerotia development [22,32-34]

In order to manipulate efficiently secondary metabolism (to enhance production of beneficial metabolites and to control production of detrimental ones) we must understand the "molecular switch" mechanism that controls the initiation of secondary metabolism. Reaching this understanding requires a cooperative effort from genomic, proteomic, and metabolomic research. Despite advances in knowledge about the genes involved in biosynthesis and the regulation of many secondary metabolitic pathways, a detailed understanding of how secondary metabolism integrates with other metabolic and cellular processes is still not available [12,25,35-39].

Metabolomics is a powerful tool to characterize the metabolic state of the cell and to discover new metabolites and biochemical pathways [40]. Volatiles, one important group of cellular metabolites, represent a significant portion of the metabolome. Many organic compounds are present in the volatile phase including acids, alcohols, aldehydes, esters, short chain fatty acids, lipid oxides, terpenes, and phenolics. In this study we applied volatile profiling analysis for gaining rapid access to information on intracellular metabolism in the fungus. Specifically, we examined carbon flow in the presence or absence of secondary metabolism in $A$. parasiticus using wild type and mutant strains carrying genetic defects specifically in aflatoxin biosynthesis and in VeA, a global regulator of secondary metabolism. The volatile metabolites generated by the fungus were analyzed using solid phase microextraction - gas chromatography/mass spectrometry (SPME-GC/MS). This analytical approach is a non-invasive and solvent-free absorption technique that is used in analysis of volatile compounds from the headspace above the sample [41]; the technique has been 
widely employed in volatile analysis (profiling) of plants, yeast, and bacteria because it is accurate, sensitive, and robust [41-48]. To conduct this procedure, the outer polymer coating of a fused silica fiber absorbs volatiles from the headspace in the growth environment; the volatiles are then desorbed in the hot GC inlet and chromotographed in the usual manner. The separated compounds are subsequently identified by mass spectrometry.

Using SPME-GC/MS volatile profiling analysis we demonstrated that a genetic block in aflatoxin biosynthesis or disruption of the global regulator $v e A$ re-directs intracellular carbon flow. Specifically, we observed that VeA negatively regulates catabolism of branched chain amino acids and the synthesis of ethanol in $A$. parasiticus; these metabolic changes were mediated at least in part at the transcriptional level. We also showed that volatile metabolites generated under the control of VeA may participate in the molecular machinery that regulates conidiation and sclerotia formation.

\section{Results}

\section{Profiling of volatile compounds in A. parasiticus SU-1}

We withdrew samples from cultures at regular intervals during growth and analyzed volatiles following a 1 to $2 \mathrm{~h}$ equilibration period; this sampling method resulted in stable and reproducible measurements. We also analyzed volatile compounds in a control injection that originated from the SPME fiber, the glass vial, and the screw cap and valve; these volatiles were excluded from the analysis of volatiles detected in the fungal culture headspace. The relative amounts of volatiles produced in culture were assessed based on instrument response [42]; these compounds were designated as possible, or putative, fungal metabolites since they could be identified by comparison with a mass spectrum library. Since the $A$. parasiticus strains used in the study (Table 1) did not differ significantly in growth rate in liquid YES medium (Additional File 1, Figure S1), the relative intensity change of all masses detected was also related to the levels of the compounds produced in culture. Compounds with no match in the NIST mass spectrum library were defined as

Table 1 Strains used in the study

\begin{tabular}{|c|c|c|}
\hline Strain & Genotype & Source \\
\hline $\begin{array}{l}\text { A. parasiticus SU-1 (ATCC } \\
56775 \text { ) }\end{array}$ & wild type & ATCC \\
\hline A. parasiticus ATCC 36537 & ver-1 wh & ATCC \\
\hline $\begin{array}{l}\text { A. parasiticus } \triangle v e A \\
\text { (TJW35.21) }\end{array}$ & $\begin{array}{l}\text { ver-1 wh pyrG } \triangle \text { veA:: } \\
\text { pyrG }\end{array}$ & $\begin{array}{l}\text { Calvo et al., } 2004 \\
{[31]}\end{array}$ \\
\hline $\begin{array}{l}\text { A. parasiticus AFS10 (ATCC } \\
\text { 24690) }\end{array}$ & aflR & $\begin{array}{l}\text { Cary et al., } 2002 \\
\text { [32] }\end{array}$ \\
\hline A. parasiticus B62 & niaD nor-1 br-1 & ATCC \\
\hline A. nidulans FGSC4 & wild type & FGSC \\
\hline
\end{tabular}

unknown. Ethanol levels produced by the fungus in culture were compared to standards. Thus, all volatiles detected fell into one of three categories: 1) known compounds identified with standards (ethanol); 2) putative compounds identified by a match in NIST mass spectrum library; and 3) unknown compounds.

The volatile profile of SU-1 grown for $72 \mathrm{~h}$ in liquid YES (aflatoxin inducing conditions) in the dark revealed 24 putative fungal metabolites and 25 unknown compounds (Additional File 2, Figure S2). These volatiles could be divided into several classes of chemical

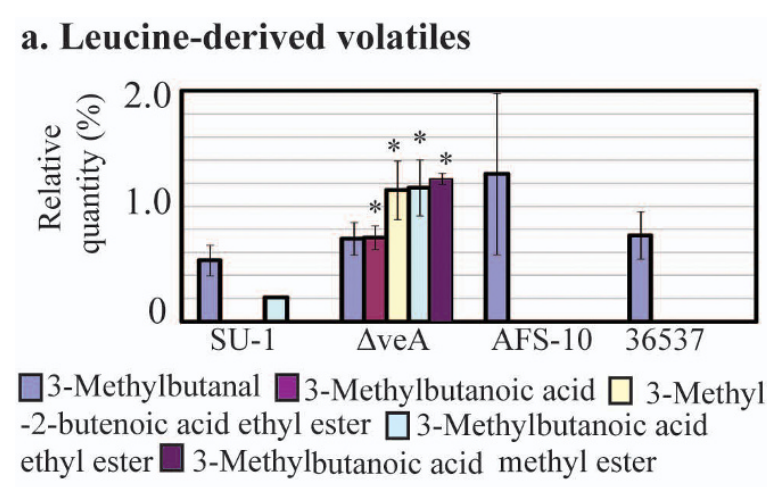

b. Isoleucine-derived volatiles

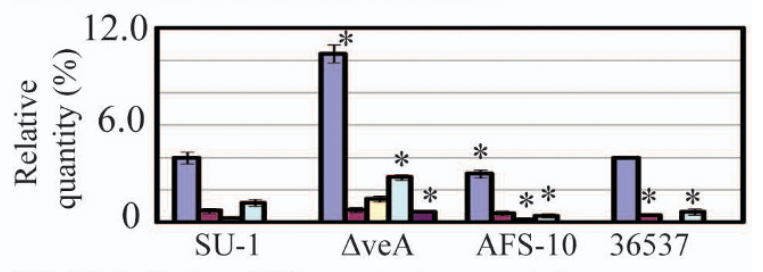

$\square$ 2-Methylbutanol $\square$ 2-Methylbutanal $\square$ 2-Methyl -2-butenoic acid ethyl ester $\square$ 2-Methylbutanoic acid ethyl ester $\square$ 2-Methylbutanoic acid methyl ester

\section{c. Valine-derived volatiles}

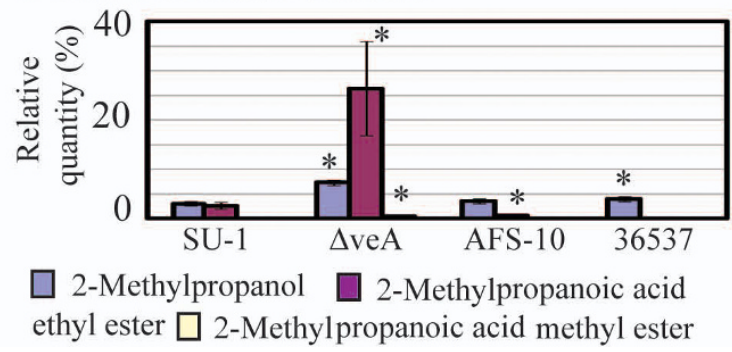

Figure 1 Branched chain amino acid-derived volatiles generated by $A$. parasiticus strains grown in YES for $72 \mathrm{~h}$ in the dark. Conidiospores were inoculated into $100 \mathrm{ml}$ of liquid YES medium at $10^{4} / \mathrm{ml}$ and the cultures were grown at $30^{\circ} \mathrm{C}$, with shaking at150 rpm, in the dark for $72 \mathrm{~h}$. Volatiles were analyzed as described in Methods. *, statistically significant difference as compared with SU-1, $\mathrm{P}<0.01$ 


\section{a. Leucine-derived volatiles}

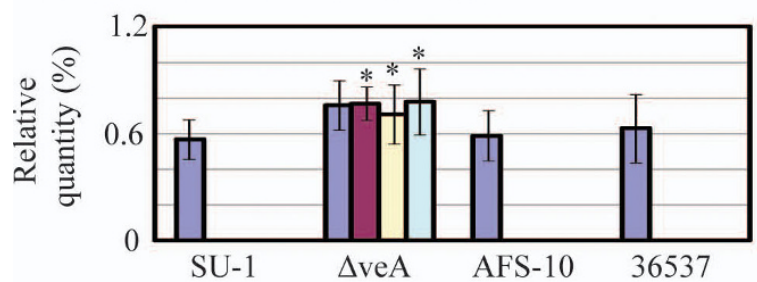

3-Methylbutanal $\square$ 3-Methyl-2-butenoic acid ethyl ester $\square$ 3-Methylbutanoic acid ethyl ester $\square$ 3-Methyl butanoic acid methyl ester

\section{b. Isoleucine-derived volatiles}

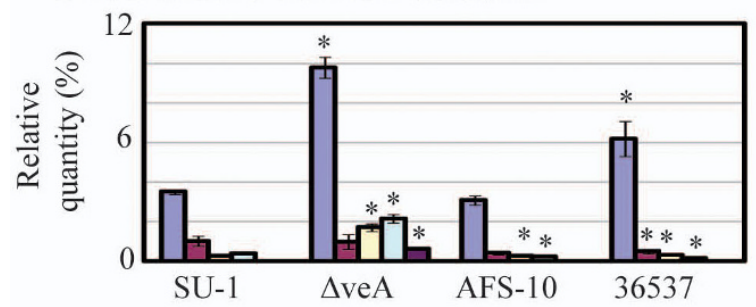

2-Methylbutanol $\square$ 2-Methylbutanal $\square$ 2-Methyl -2-butenoic acid ethyl ester $\square$ 2-Methyl butanoic acid ethyl ester $\square$ 2-Methylbutanoic acid methyl ester

\section{c. Valine-derived volatiles}

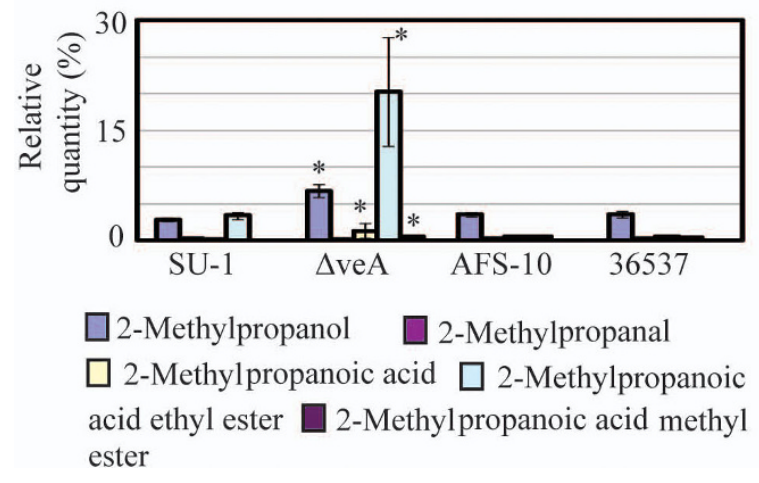

Figure 2 Branched chain amino acid-derived volatiles generated by $A$. parasiticus strains grown in YES for $72 \mathrm{~h}$ in the light. Conidiospores were inoculated into $100 \mathrm{ml}$ of liquid YES medium at $10^{4} / \mathrm{ml}$ and the cultures were grown at $30^{\circ} \mathrm{C}$, with shaking at $150 \mathrm{rpm}$, in the light for $72 \mathrm{~h}$. Volatiles were analyzed as described in Methods. *, statistically significant difference as compared with SU-1, P $<0.01$

compounds. The largest class of putative fungal metabolites included compounds derived from intermediates in metabolism of branched chain amino acids (leucine, isoleucine, and valine; see Additional File 3, Figure S3) and esters. Additional classes of compounds included alcohols (1-butanol, 1-propanol, and ethanol), lipid-derived volatiles (2-methylfuran and 1,1-diethoxy-ethane), aldehydes (formaldehyde), and organic acids (acetic acid).
The relative quantities of volatiles derived from metabolism of branched chain amino acids in the dark and light were similar (Figure 1, 2). However, we observed differences in number of leucine- and valine-derived volatiles (but not isoleucine-derived volatiles) that were produced in the light versus dark. The number and relative quantities of branched chain amino acid-derived volatiles detected in the light in YES were higher at $72 \mathrm{~h}$ as compared with $48 \mathrm{~h}$ (Additional File 4, Figure S4).

We compared volatiles generated by $A$. parasiticus SU-1 grown for $72 \mathrm{~h}$ in the light in GMS (chemically defined medium, contains glucose) to those generated in YES. In GMS, the fungus produced a lower number of compounds of all classes of volatiles identified (including volatiles derived from branched chain amino acids and lipids) than in YES (not shown).

\section{A genetic block in aflatoxin biosynthesis affects the volatile profile}

We compared volatiles produced by $A$. parasiticus SU-1 (wild type) and A parasiticus strains impaired in aflatoxin biosynthesis, AFS10 and 36537, grown in a rich medium (YES) for $72 \mathrm{~h}$ in the dark. Aflatoxin synthesis is blocked in AFS10 (gene disruption in a positive pathway regulator, aflR; no aflatoxin enzymes or aflatoxin are synthesized) and in A. parasiticus ATCC36537 that carries a mutation in the aflatoxin pathway gene, ver-1 (accumulates the pathway intermediate versicolorin $\mathrm{A}$ ). In the dark, AFS10 and 36537 generated similar relative quantities of 3-methylbutanal, a presumable intermediate in leucine metabolism, as compared to the wild type strain SU-1 (Figure 1a). However, no 3-methylbutanoic acid ethyl ester was produced by these two mutants (Figure 1a). None of the strains studied produced 3-methylbutanol as well.

All studied strains generated 2-methylbutanol, a putative derivative of isoleucine catabolism (Figure 1b). Nonetheless, the ethyl and methyl esters of the corresponding 2-methylbutanoic acid (2-methylbutanoic acid ethyl ester and 2-methylbutanoic acid methyl ester) were produced by the mutants in less quantity as compared with SU-1.

Accumulation of 2-methylpropanoic acid ethyl ester, a derivative of valine metabolism, was significantly reduced in AFS10 and 36537 as compared to the wild type SU-1 (Figure 1c). All strains, SU-1, AFS10, and 36537, generated 2-methylpropanol.

\section{Disruption of veA enhances accumulation of metabolites in branched chain amino acid catabolism}

The volatile profile produced by $\Delta v e A$ was significantly different than the profile of SU-1 (the wild type) and ATCC 36537 (genetic control for $\Delta v e A$ ). A. parasiticus $\Delta v e A$ generated significantly higher quantities of 
metabolites (relative to SU-1 and 36537) derived from catabolism of the branched chain amino acids leucine, isoleucine, and valine in the dark and in the light (Figure 1, 2 ). For instance, quantities of the branched chain alcohols 2-methylbutanol, and 2-methylpropanol were doubled in $\Delta v e A$. Ethyl and methyl esters derived from branched chain amino acids (derived presumably from leucine, isoleucine, and valine) increased up to 10 fold (and higher for several compounds) as compared with SU-1 and 36537. Four esters were unique to $\Delta v e A$ (Additional File 5, Figure S5). One of these, 2-methylbutanoic acid methyl ester is found in the aroma of gooseberry [49], which may explain the observed fruity smell of $\Delta v e A$ cultures.

Interestingly, more than 2 fold higher quantities of ethyl acetate and acetic acid were also detected in $\Delta v e A$ (Additional File 2, Figure S2) in comparison to 36537 and SU-1.

\section{Feeding A. parasiticus $\Delta v e A$ with leucine, isoleucine, and valine}

We determined that YES medium contains low levels of 2-methylbutanal, 3-methylbutanal, and 2-methylpropanal (not shown), which serve as precursors to synthesis of the corresponding branched chain alcohols. To examine whether the branched chain alcohols and esters generated in elevated quantities by $A$. parasiticus $\Delta v e A$ relate to catabolism of the branched chain amino acids leucine, isoleucine and valine by the fungus, these amino acids were added to $48 \mathrm{~h}$ old cultures of A. parasiticus $\Delta v e A$ at a final concentration of $0.03 \mathrm{M}$ and the volatiles were analyzed after $18 \mathrm{~h}$. Methionine (0.03 $\mathrm{M}$ final concentration) was added to a separate flask as a control. Feeding with leucine increased formation of the esters corresponding to leucine catabolism; however, formation of 3-methylbutanol, an expected product of leucine catabolism, was not detected either with or without addition of leucine (Figure 1a, Figure 3a). Added isoleucine and valine significantly (several fold) increased production of the expected corresponding esters and alcohols (2-methylbutanol, and 2-methylpropanol) (Figure 3b, c). Feeding with amino acids also elevated production of certain non-corresponding volatiles. For example, addition of valine increased accumulation of 3-methylbutanoic acid ethyl ester, a product of leucine catabolism. Addition of isoleucine and methionine increased formation of the products of valine catabolism including 2-methylpropanoic acid ethyl ester.

\section{Disruption of veA increases ethanol production by A. parasiticus $\triangle v e A$}

It was shown previously that aspergilli can produce ethanol [50]. In that study, an inverse regulatory relationship between aflatoxin and ethanol accumulation was demonstrated. Aflastatin A, an inhibitor of aflatoxin

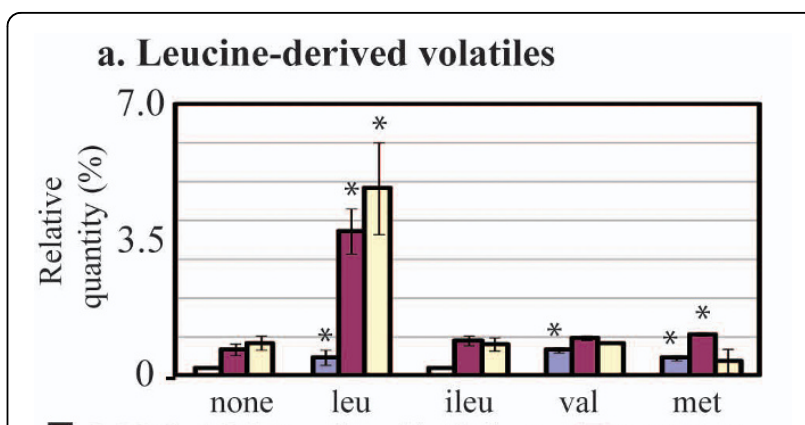

3-Methyl-2-butenoic acid ethyl ester $\square$ 3-Methyl butanoic acid ethyl ester $\square$ 3-Methylbutanoic acid methyl ester

\section{b. Isoleucine-derived volatiles}

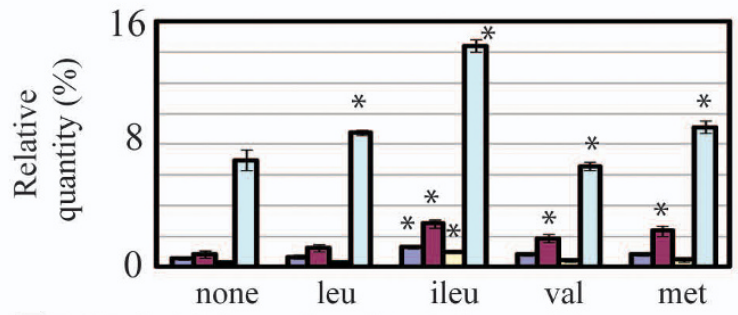

2-Methyl-2-butenoic acid ethyl ester $\square$ 2-Methyl butanoic acid ethyl ester $\square$ 2-Methylbutanoic acid methyl ester $\square$ 2-Methylbutanol

\section{c. Valine-derived volatiles}

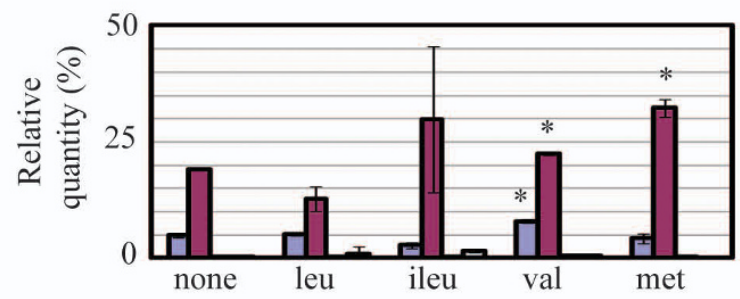

2-Methylpropanol $\square$ 2-Methylpropanoic acid ethyl ester $\square$ 2-Methylpropanoic acid methyl ester 2-Methylpropanoic acid

Figure 3 Effect of amino acid feeding on volatile production by $A$. parasiticus $\Delta v e A$ grown in the dark. Conidiospores were inoculated into $100 \mathrm{ml}$ of liquid YES medium at $10^{4} / \mathrm{ml}$ and the cultures were grown at $30^{\circ} \mathrm{C}$, with shaking at $150 \mathrm{rpm}$, in the dark for $48 \mathrm{~h}$. Then the amino acids were added and volatiles were analyzed after $18 \mathrm{~h}$ of additional incubation as described in Methods.

production, was shown to inhibit aflatoxin biosynthesis and concurrently to inhibit ethanol catabolism at the transcriptional level thus resulting in an increase of ethanol accumulation by $A$. parasiticus; glucose consumption also increased [50,51].

We demonstrated that in YES, A. parasiticus strains including SU-1, B62 (nor-1 mutant, accumulates the 


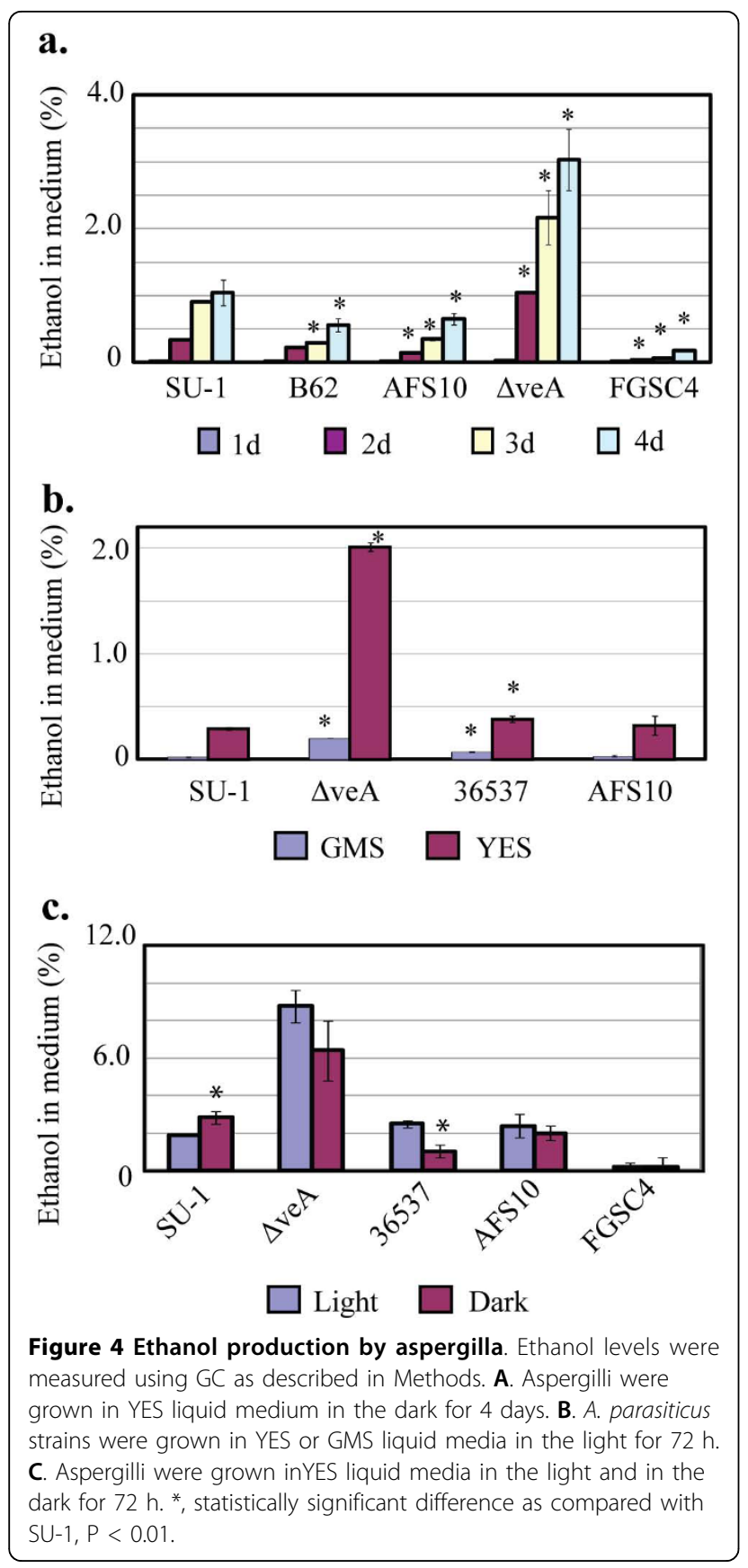

pathway intermediate norsolorinic acid), AFS10, and $\Delta v e A$ produced significantly higher quantities of ethanol than $A$. nidulans FGSC4 at each time point tested (the experiment was performed in the dark for 4 days) (Figure 4a). In the chemically defined medium GMS, all A. parasiticus strains tested (SU-1, AFS10, 36537, and $\triangle v e A)$ generated several fold lower quantities of ethanol as compared with YES medium (Figure 4b). Light did not influence ethanol production by either strain of A. parasiticus (Figure 4c). Feeding with leucine (as described above) did not significantly affect production of ethanol by the wild type SU-1 (not shown).

A genetic block in aflatoxin biosynthesis in AFS10 or in 36537 resulted in a decreased formation of ethanol by these mutant strains in comparison to SU-1. However, disruption of $v e A$ resulted in 3 to 4 fold higher levels of ethanol as compared to SU-1, or 36537 (Figure 4a, b, c) under all conditions tested; the concentration of ethanol in the $\Delta v e A$ culture medium ranged from 2 to $8 \%$. Feeding with leucine and valine (as described above) did not significantly affect production of ethanol by $\Delta v e A$ (not shown). However, isoleucine feeding resulted in a slight inhibition of ethanol production (not shown).

\section{Volatiles produced by $A$. parasiticus $\Delta v e A$ affect conidiospore and sclerotia formation}

Disruption of $v e A$ results in developmental defects (blocks asexual conidiation in the dark and sclerotia formation [32,52]). We previously showed that fungal volatiles play a role in the control of secondary metabolism [53]. To test whether the volatiles produced by $\Delta v e A$ participate in the molecular machinery that regulates aflatoxin biosynthesis and asexual conidiation, we grew A. parasiticus B62 (accumulates the red colored aflatoxin intermediate norsolorinic acid along the colony margin) on agar medium in the presence of volatiles generated by $\Delta v e A$. We observed an approximately $35 \%$ to $55 \%$ reduction in conidiation in B62 after exposure to $\Delta v e A$ volatiles for 5 days (Figure 5). Accumulation of norsolorinic acid was not affected (not shown).

To analyze the effect of $\Delta v e A$ volatiles on sclerotia formation, $A$. parasiticus SU-1 and ATCC 36537 were grown on coconut or YGT agar media (both media were previously shown to induce sclerotia formation $[22,32])$ in the dark in the presence of $\Delta v e A$ volatiles (see Methods). A. parasiticus SU-1 grown on coconut agar medium for 9 days demonstrated an approximately 30 to $40 \%$ decrease in the number of sclerotia in the presence of $\Delta v e A$ volatiles (Table 2). However, no significant effect on the number of sclerotia formed on YGT was observed (not shown). Under all conditions tested, sclerotia were black in color and were able to produce colonies after harvest followed by inoculation onto YES agar medium. Interestingly, SU-1 conidiospores that developed on coconut medium in the presence of $\Delta v e A$ volatiles for 17 days were dark brown, whereas conidiospores developed under SU-1 volatiles were dark green indicating that volatiles also may affect biosynthesis of conidial pigment.

\section{Analysis of transcript accumulation for branched chain} amino acid aminotransferase and alcohol dehydrogenase The first reaction in the catabolism of branched chain amino acids is catalyzed by a branched chain amino 

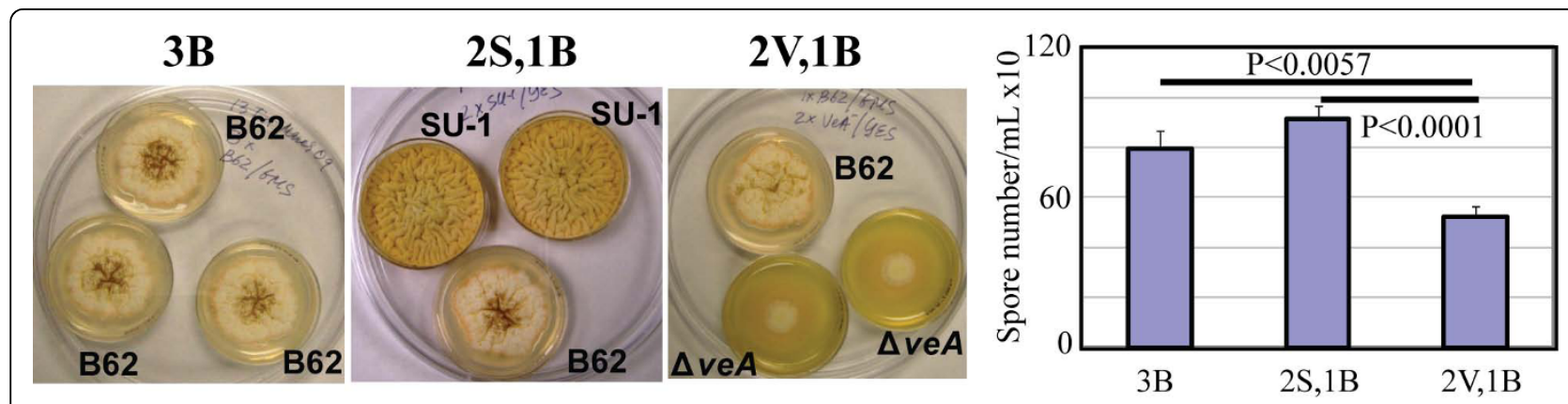

\section{B: $A$. parasiticus B62, S: $A$. parasiticus $\mathrm{SU}-1, \mathrm{~V}: A$. parasiticus $\Delta$ veA}

Figure 5 Effect of fungal volatiles on A. parasiticus B62 sporulation. Spores were center inoculated on agar media and grown for 5 days at $30^{\circ} \mathrm{C}$ in the dark. B62 was grown on GMS while SU-1 and $\triangle$ veA were grown on YES agar medium. Small Petri dishes containing colonies (no lids) were arranged within large Petri dishes as shown above. B62 conidia are represented by dark brown dots in the center of colonies.

acid aminotransferase that forms a 2-ketoacid; this reaction controls the flow of carbon through the catabolic pathway [54]. The resulting 2-ketoacid can then be transformed into a branched chain alcohol (after decarboxylation in the presence of 2-keto acid decarboxylase), and/or into ethyl or methyl esters (see schematic in Additional File 3, Figure S3). In order to examine possible mechanisms that generate the observed elevation in the accumulation of catabolic products of branched chain amino acids, the expression of branched chain amino acid aminotransferase gene expression was analyzed. The genome of $A$. flavus, a close relative of $A$. parasiticus $[12,14]$, contains two genes (AFLA_113800 and AFLA_044190) that encode proteins that exhibit a high percentage identity with the Saccharomyces cerevesiae branched chain amino acid aminotransferases BAT1 (mitochondrial) and BAT2 (cytosolic). AFLA_113800 exhibits $61 \%$ identity to $S$. cerevisiae branched chain amino acid aminotransferase BAT1 and $60 \%$ identity to BAT2 (Additional File 6, Figure S6). AFLA_044190 is $43 \%$ identical to BAT1 and $44 \%$ identical to BAT2. The expression of AFLA_113800 and AFLA_044190 was detected in SU-1 and 36537 (Figure 6). Interestingly, in $\triangle v e A$ the expression levels for these genes were approximately 2 fold higher at $40 \mathrm{~h}$ as compared with SU-1 and 36537 (Figure 6); at this time point, aflatoxin biosynthesis peaks in SU-1. However, there were no significant differences in the relative concentrations of branched amino acids in SU-1, $\Delta v e A, 36537$, and AFS10 cultures grown for $72 \mathrm{~h}$ in YES (not shown).

Since we observed a significant increase in the level of ethanol accumulation in $\Delta v e \mathrm{~A}$, we analyzed the expression of a gene encoding alcohol dehydrogenase, AFLA_048690. This gene exhibits the highest sequence identity (57\%) with $S$. cerevesiae adh1, a gene that encodes an alcohol dehydrogenase (Additional File 7, Figure S7). adh1 accounts for the majority of alcohol dehydrogenase activity in baker's yeast and primarily is responsible for ethanol formation [55]. AFLA_048690 also exhibits 50 to $55 \%$ identity to the yeast genes $a d h$ 2,3 , and 5 . The yeast genes $a d h 1,2,3$, and 5 are also known to participate in the catabolism of amino acids to produce branched chain alcohols [56]. In $\Delta v e A$ the expression level for AFLA_048690 was significantly higher at $40 \mathrm{~h}$ as compared with SU-1 and 36537 (Figure 6); the same pattern of expression was observed

Table 2 Volatiles generated by $A$. parasiticus $\Delta v e A$ reduce sclerotia production by SU-1 grown on coconut agar medium

\begin{tabular}{|c|c|c|c|c|}
\hline \multirow[t]{2}{*}{ Sclerotia developed by SU-1 on coconut agar medium; lid \# } & \multicolumn{4}{|c|}{$\begin{array}{l}\text { Volatiles were produced by aspergilli grown on YES or coconut agar } \\
\text { medium, or YES agar medium only (two lids of each) }\end{array}$} \\
\hline & $\Delta v e A$ on YES & SU-1 on coconut & SU-1 on YES & $\begin{array}{l}\text { YES } \\
\text { only }\end{array}$ \\
\hline 1 & 231 & 397 & 252 & 493 \\
\hline 2 & 231 & 364 & 314 & 258 \\
\hline 3 & 232 & 618 & $n \backslash e$ & 307 \\
\hline
\end{tabular}

Three $60 \times 15 \mathrm{~mm}$ Petri dish lids were placed inside a large $150 \times 15 \mathrm{~mm}$ Petri dish as described in Methods. For sclerotia development $10^{4}$ spores of $A$. parasiticus SU-1 were center inoculated onto one lid that contained coconut agar medium. For volatiles generation, two other lids contained either $\triangle$ veA inoculated on YES agar medium, or SU-1 inoculated on coconut agar medium (control), or SU-1 inoculated on YES agar medium (control), or YES agar medium only (control). The cultures were grown in the dark at $30^{\circ} \mathrm{C}$ for 9 days. The experiment was performed in triplicate. The number of sclerotia developed by SU-1 grown on coconut agar medium per plate is presented. $n \backslash e$, not estimated. 


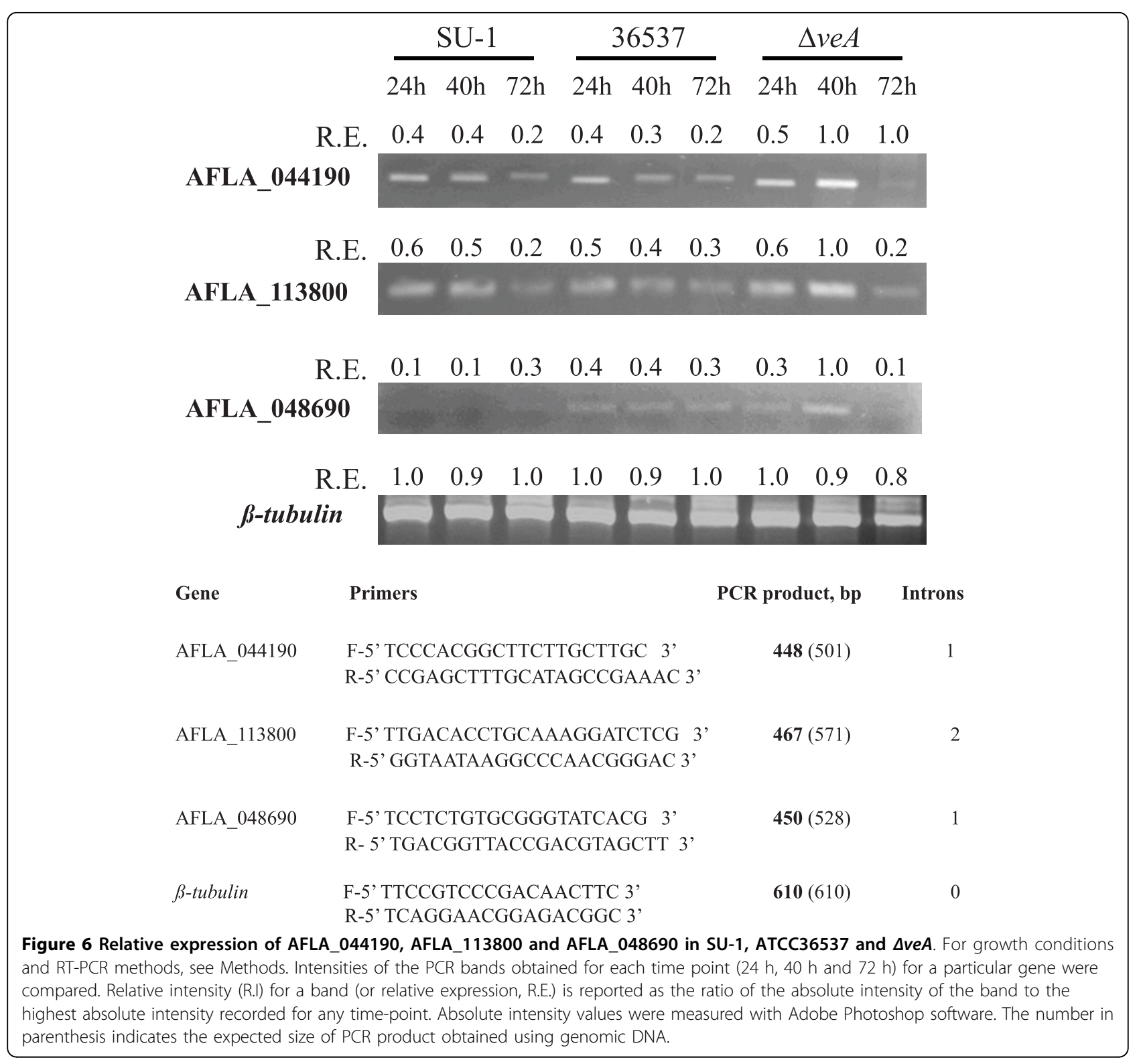

for the putative branched chain amino acid transferases AFLA_044190 and AFLA_113800 (see above, Figure 6). These results strongly suggest that VeA negatively regulates the formation of branched chain amino acidderived volatiles and ethanol as the cells trigger secondary metabolism.

\section{VeA is a positive regulator of mitochondrial and peroxisomal $\beta$-oxidation}

$\beta$-oxidation of fatty acids is one source that supplies precursors for polyketide biosynthesis; in addition, $\beta$ oxidation of odd number fatty acids generates propionyl-CoA that can affect the activity of a polyketide synthase involved in sterigmatocystin biosynthesis
$[57,58]$, thus presumably contributing to the $\Delta v e A$ phenotype. Propionate is also a product of catabolism of several amino acids, including valine and isoleucine. The inability of null mutants $\triangle v e A$ and $\triangle l a e A$ to grow on peanut and maize seeds [59] may be explained by the failure of the mutants to metabolize host lipids due to defects in $\beta$-oxidation.

We focused our attention on the genes echA and fox $A$, which encode, respectively, a short chain enoyl-CoA hydratase (EchA) involved in $\beta$-oxidation in mitochondria, and a multifunctional enzyme FoxA (possesses enoyl-CoA hydratase and hydroacyl-CoA dehydrogenase activities) involved in $\beta$-oxidation of long chain fatty acids in peroxisomes; these genes previously were 


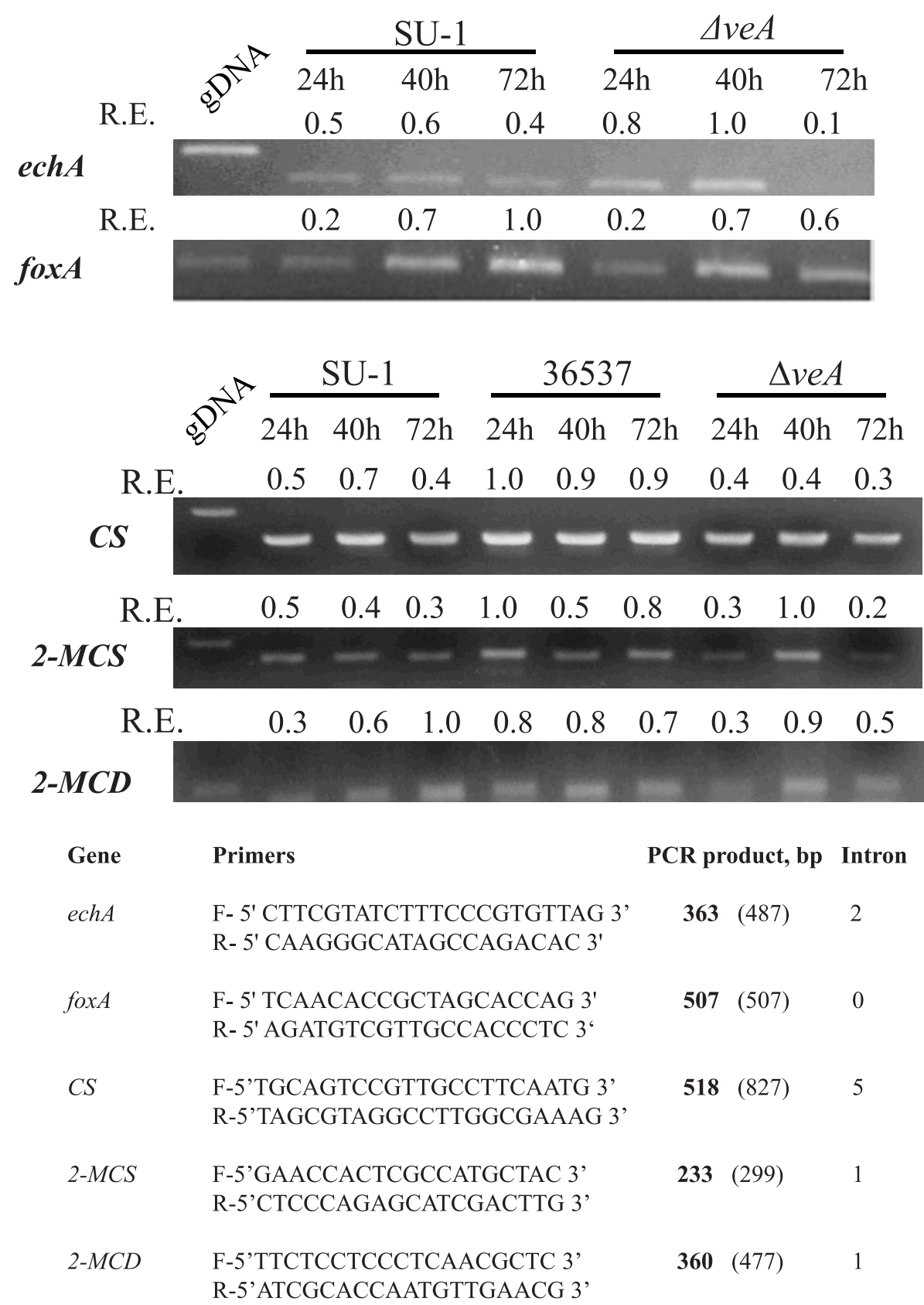

Figure 7 Transcript analysis of genes involved in the methylcitrate cycle and $\beta$-oxidation of fatty acids in A. parasiticus strains. For growth conditions and RT-PCR methods, see Methods. R.E., relative expression was calculated as shown in Figure 6. CS, citrate synthase AFLA_007020; 2-MCS, 2-methylcitrate synthase AFLA_049290; 2-MCD, 2-methylcitrate dehydratase AFLA_056350; echA, a short chain enoyl-CoA hydratase AFLA_043610; foxA, an enoyl-CoA hydratase/hydroacyl-CoA dehydrogenase AFLA_041590. The number in parenthesis indicates the expected size of PCR product obtained using genomic DNA.

shown to be involved in $\beta$-oxidation in $A$. nidulans $[25,38]$. A BLAST search using sequences of $A$. nidulans fox $A$ and echA identified two homologous genes in the genome of $A$. flavus, a close relative of $A$. parasiticus $[12,14]$. AFLA_041590 has $81 \%$ identity to A. nidulans foxA; AFLA_043610 has $83 \%$ identity to $A$. nidulans echA. To analyze transcript accumulation in
A. parasiticus, primers were designed based on the A. flavus gene sequences (Figure 7).

Expression of both genes in the wild type SU-1and in $\triangle v e A$ increased from $24 \mathrm{~h}$ to $40 \mathrm{~h}$ of growth (Figure 7 ). By $72 \mathrm{~h}$ of growth we observed a decline in echA transcript accumulation in SU-1 and $\Delta v e A$; however the decrease in $\triangle v e A$ was more severe than in SU-1. 
By $72 \mathrm{~h}$ of growth, transcript accumulation of fox $A$ in SU-1 continued to increase, whereas in $\triangle v e A$ accumulation of the fox $A$ transcript declined slightly.

The methylcitrate cycle is one biochemical pathway for propionate metabolism in fungi [60-63]. We hypothesized that impairment of methylcitrate cycle would increase formation of corresponding valine- and leucine-derived esters. We examined transcript accumulation of the first two genes of the methylcitrate cycle, 2-methylcitrate synthase (2-MCS) and 2-methylcitrate dehydratase (2-MCD), in A. parasiticus strains (Figure 7 ); we also compared their pattern of accumulation with transcript accumulation of citrate synthase. We detected transcripts for all three genes in all strains tested. However, in $\triangle v e A$, transcripts for 2-MCS and 2-MCD increased at $30 \mathrm{~h}$ and declined by $40 \mathrm{~h}$, in contrast to the wild type SU-1, which showed a slight decrease in transcript accumulation for 2-MCS from $24 \mathrm{~h}$ to $40 \mathrm{~h}$. These data suggest that the 2-methylcitrate cycle is not impaired in $\Delta v e A$.

\section{Discussion}

A metabolomics approach previously was used to link complex biochemical and cellular functions to genomics in plants and yeast [45,64-66]. However, relatively few (and incomplete) metabolomics studies have been reported for filamentous fungi [3,67-70]. Volatile compounds represent a significant portion of the metabolome and, as has been demonstrated in our study, they provide information on the real-time metabolic changes that occur within the fungal cell; most importantly from a practical stand point, this approach does not require quantification of the metabolites or cell disruption. We show that SPME-GC/MS is a sensitive, fast, and accurate approach to study changes in volatile compounds generated by the filamentous fungi.

Our studies demonstrate that $A$. parasiticus produces a variety of volatile organic compounds including several classes of intermediates and products associated with catabolism of the branched chain amino acids (leucine, isoleucine, and valine) and lipids; alcohols, organic acids, esters, and aldehydes were also detected. Our data suggest that $A$. parasiticus catabolizes free branched chain amino acids (endogenously synthesized, or exogenously added); these may either enter the catabolic pathway directly, or they could be used as a carbon source, thus producing detectable levels of metabolic cross talk.

2-ketoacids are also synthesized de novo as late intermediates in branched chain amino acid biosynthesis pathways; they can be converted to the corresponding alcohols and esters. Our feeding studies provide evidence that fungal volatile compounds originate from branched chain amino acids catabolism; however, we can not rule out the possibility that 2 -ketoacids generated de novo through biosynthetic pathway serve as an additional precursor for volatile synthesis.

We also demonstrate that the number of volatile compounds produced in culture depends on the composition of the growth medium, the presence or absence of light, and also on the status of secondary metabolism in the fungal cell. A genetic block in aflatoxin biosynthesis in AFS10 and ATCC 36537 resulted in a decrease in formation of isoleucine- and valine-derived acids and esters; intermediates in leucine catabolism (except for 3methylbutanal) were barely detected. These data support the idea that secondary metabolism (aflatoxin biosynthesis) is an integrated part of the cellular metabolism.

Our data show that disruption of secondary metabolic pathways in $\triangle v e A$ correlate with dramatic changes in carbon flow through primary metabolic pathways. The most significant metabolic changes were observed in catabolism of branched chain amino acids and formation of ethanol in $A$. parasiticus; the data strongly suggest that VeA acts as a negative regulator of these processes at the transcriptional level. Based on previous and current work, we propose a model for the association between secondary metabolism and catabolism of branched chain amino acids and ethanol biosynthesis in A. parasiticus; the model proposes that Velvet A plays a key regulatory role in the coordination of carbon flow through these metabolic processes (Figure 7).

How can one explain the changes in carbon flow observed in $\Delta v e \mathrm{~A}$ ? Under conditions studied (liquid shake culture, no development occurs) disruption of $v e A$ is thought to impair most if not all of secondary metabolism [32,52]. Thus it is reasonable to suggest that the cell must re-structure and re-direct its metabolism and carbon flow in order to maintain cellular homeostasis. An increase in production of ethanol and branched chain alcohols and esters may serve as a compensatory mechanism to maintain cellular redox balance and to promote carbon removal from the cell. Our data suggest that, at least at the transcription level, accumulation of acetyl-CoA and propionyl-CoA in SU-1 are balanced by channeling through polyketide biosynthesis, the methylcitrate cycle, and ethanol formation. At $40 \mathrm{~h}$, the $\Delta v e A$ strain, which is aberrant in secondary metabolism, compensates for the increase in accumulation of propionylCoA by increasing methylcitrate cycle activity as well as the formation of ethyl-propionate and corresponding esters. Acetyl-CoA is re-directed through ethanol biosynthesis. The block in secondary metabolism in $\Delta v e A$, also directs carbon flow through formation of branched chain acyl-CoA-derived alcohols, acids and esters. At 72 $\mathrm{h}$, these compensatory mechanisms in $\Delta v e A$ likely discontinue resulting in the accumulation of acetyl-CoA and propionyl-CoA, and in the activation of a feed-back inhibition mechanism that affects $\beta$-oxidation both in 
mitochondria and peroxisomes. In this scenario, VeA controls $\beta$-oxidation indirectly through accumulation of acetyl-CoA and propionyl-CoA. However, whether the effect of VeA on gene transcription is mediated directly through protein-protein interactions, indirectly through intracellular biochemical changes, or both, remains to be elucidated.

One alternative explanation for the observed changes in carbon flow in $\Delta v e \mathrm{~A}$ relates to studies on the biosynthesis of polyketide antibiotics by Streptomyces spp. The polyketides virginiamycin, tautomycin, mananumycin, butyrolactols, and antraquinones are generated by condensation of starter units including isobutyryl-CoA, isovaleryl-CoA, and 2-methylbutyryl-CoA; the latter are derived from catabolism of the branched chain amino acids valine, leucine, and isoleucine respectively $[71,72]$. We propose that Aspergillus spp. synthesize polyketide(s) using branched acyl-CoA as precursors that originate through catabolism (or biosynthesis) of branched chain amino acids and or $\beta$-oxidation of fatty acids, and that $v e A$ positively regulates this biosynthetic pathway. Maggio-Hall et al. [39] provided evidence that mitochondrial $\beta$-oxidation of fatty acids and catabolism of branched chain amino acids utilize the same acyl-CoA dehydrogenase encoded by $s c d A$. We hypothesize that, in the presence of VeA, $\beta$-oxidation of fatty acids and catabolism of branched chain amino acids occur in mitochondria and provide the necessary starter units for biosynthesis of polyketides, similar to the situation observed in Streptomyces spp. In $\Delta v e \mathrm{~A}$, the biosynthesis of these polyketide(s) is blocked, which causes a shift in metabolism to stimulate accumulation of branched chain alcohols and branched chain esters (Figure 8). The regulatory feed back mechanisms underlie the decline in $\beta$-oxidation of fatty acids. Interestingly, valine is synthesized also in the mitochondrion [73]. Alternatively, A. parasiticus produces other secondary metabolites derived from branched chain amino acids. Future work is focused on testing the hypothesis stated above.

$\mathrm{VeA}$ is a global regulator of morphogenesis and secondary metabolism in Aspergillus spp [12,24,32,52]; this gene is involved in transcriptional regulation of several hundred genes [74]. Our work provides additional mechanistic details about the molecular machinery by which VeA regulates gene expression and therefore conidiation and sclerotia formation. We demonstrated that deletion of $v e A$ results in accumulation of volatile compounds with biological activity, which in turn, participate in the regulation of developmental processes. What is the role of LaeA that forms a protein complex with $\mathrm{VeA}$ in this regulation? Future studies are necessary to better understand the mechanisms that underlie this phenomenon.

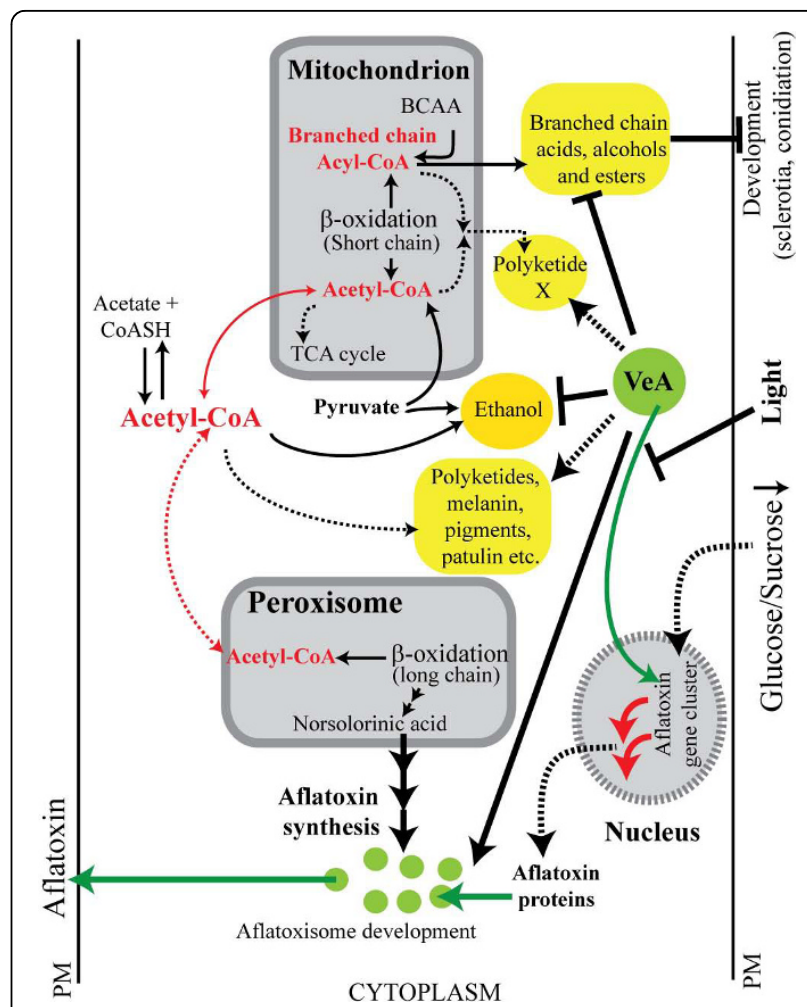

Figure $8 \mathrm{VeA}$ controls intracellular carbon flow in Aspergillus parasiticus. The schematic illustrates compartmentalization of biosynthesis of secondary metabolites, branched chain amino acid catabolism, and biosynthesis of ethanol in Aspergillus. Acetyl-CoA is produced in the mitochondrion, the peroxisome and in the cytoplasm; acetyl-CoA is the precursor of aflatoxin and other secondary metabolites [4]. Early steps in aflatoxin biosynthesis occur in peroxisomes [22]; the middle and late steps take place in aflatoxisomes $[4,12]$. The biosynthesis and catabolism of branched chain amino acids occur in the mitochondrion; branched chain acylCoAs serve as the precursors of branched chain acids, branched chain alcohols and branched chain esters. Acyl-CoA and acetyl-CoA serve as the precursors of the unknown polyketide $X$. Ethanol is produced through nonoxidative decarboxylation of pyruvate followed by conversion of acetaldehyde to ethanol by alcoholdehydrogenase; acety-CoA may also be converted to ethanol. VeA negatively regulates branched chain amino acid catabolism and ethanol biosynthesis. In addition, VeA is a positive regulator of $\beta$-oxidation of fatty acids in mitochondria and peroxisomes during the late stages of stationary phase. When secondary metabolism is blocked in $\Delta v e A$, carbon flow is re-directed to elevated ethanol production and branched chain amino acidderived volatiles. Overall, VeA is "a master-coordinator", which plays a role in regulation of carbon flow through metabolic processes (primary and secondary) in different cellular compartments. Known metabolic and regulatory pathways are shown by solid lines; hypothesized pathways are indicated by dashed lines. Abbreviations: BCAA, branched chain amino acids; PM, plasma membrane.

\section{Conclusions}

1) SPME-GC/MS volatile profiling analysis is a powerful approach to identify intracellular metabolic changes and the direction of carbon flow in filamentous fungi. An important practical advantage of this approach is that there is no need 
to calculate individual metabolite concentration or to disrupt the cells. 2) VeA coordinates biosynthesis of secondary metabolites with catabolism of branched chain amino acids and alcohol biosynthesis. 3) Our work provides insight on how changes in intracellular chemical development are linked to morphological development. 4) Understanding carbon flow through secondary metabolic pathways and catabolism of branched chain amino acids is essential for controlling and customizing production of natural products.

\section{Methods}

\section{Strains, growth media, and growth conditions}

The isogenic $A$. parasiticus strains used in this study were derived from SU-1 (ATCC 56775), a wild type aflatoxin producer (Table 1). AFS10 is an aflatoxin non-producing strain derived from the parent strain, SU-1; gene disruption of aflR in AFS10 blocks aflatoxin synthesis and expression of several aflatoxin genes. AFS10 was kindly provided by Dr. J. Cary [33,75]. A. parasiticus ATCC36537 (ver-1, wh1) was generated from $A$. parasiticus SU-1 by U.V. irradiation [76]. This strain accumulates the aflatoxin pathway intermediate versicolorin $\mathrm{A}$ due to a point mutation in Ver-1A at nucleotide residue 287 ( $G$ to $\mathrm{A}$ ) thus resulting in a non-functional enzyme [77]. The $v e A$ deletion strain $A$. parasiticus $\Delta v e A$ (ver-1, wh-1, pyrG, $\Delta v e A:: p y r G)$ was generated from $A$. parasiticus CS10 (ver-1, pyrG, wh-1) by a double-crossover event exchanging the pyrG selectable marker for the veA coding region [32]. CS10 was in turn generated from $A$. parasiticus ATCC36537 by spontaneous mutation using N-methyl-N'-nitro-N-nitrosoguanidine followed by enzymatic analysis [78].

YES liquid medium (contains 2\% yeast extract and 6\% sucrose; pH 5.8) was used as an aflatoxin inducing growth medium. A chemically defined glucose minimal

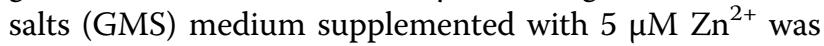
prepared as described elsewhere [79]. YGT medium $(0.5 \%$ [wt/vol] yeast extract, $2 \%[\mathrm{wt} / \mathrm{vol}]$ glucose, and 1 $\mathrm{ml}$ of trace element solution per liter of medium) was prepared as described previously [32]. Coconut agar medium was prepared as described by Mahanti et al. [22]. $10^{4}$ spores $/ \mathrm{ml}$ were inoculated into liquid medium.

To analyze the effect of fungal volatiles on aflatoxin biosynthesis and fungal development, the fungus was grown in $60 \times 15 \mathrm{~mm}$ Petri dish lids. $10^{4}$ spores were center inoculated onto the agar medium. Three lids were placed inside a larger, $150 \times 15 \mathrm{~mm}$ Petri dish as described previously [53]. This system allowed free gas and volatile exchange between colonies inside the large dish while preventing direct colony contact.

Growth of $A$. parasiticus strains was estimated by dry weight of the mycelia. Mycelia were harvested at appropriate times of growth by filtration through Miracloth (Calbiochem/EMD Biosciences, La Jolla, CA) and dried for $48 \mathrm{~h}$ at $90^{\circ} \mathrm{C}$.
Detection of aflatoxin $B_{1}, B_{2}, G_{1}, G_{2}$ and norsolorinic acid Aflatoxins in the agar medium and mycelium were extracted 3 times with $5 \mathrm{ml}$ chloroform (15 $\mathrm{ml}$ total). The extracts were dried under a stream of $\mathrm{N}_{2}$ and redissolved in $70 \%$ methanol. Aflatoxins were detected by TLC and ELISA as described by Roze at al. [80]. ELISA provided an estimation of $\mathrm{AFB}_{1}$ levels, whereas TLC enabled one to estimate levels of $\mathrm{AFB}_{1}, \mathrm{AFB}_{2}, \mathrm{AFG}_{1}$, and $\mathrm{AFG}_{2}$. Norsolorinic acid was extracted from the agar and mycelium with chloroform and then acetone, and its quantity was analyzed by TLC [81].

\section{Evaluation of conidiation}

A. parasiticus conidia were harvested and their number per colony was estimated as described by Roze et al. [80].

\section{Volatile compound analysis by SPME-GC/MS}

Sampling and volatile analysis were performed essentially as described previously [42] with minor modifications. Sample preparation and SPME analysis. Twelve $\mathrm{ml}$ of fungal culture were harvested at regular intervals and dispensed into $22 \mathrm{ml}$ clear screw cap vials equipped with Mininert ${ }^{\oplus}$ Valves (all from Supelco, Bellefonte, PA). Vials with cultures were pre-equilibrated at $30^{\circ} \mathrm{C}$ (the same temperature we used for fungal growth) in a water bath for at least $30 \mathrm{~min}$, shaking at $50 \mathrm{rpm}$, before headspace gases were sampled at $30^{\circ} \mathrm{C}$. A $65 \mu \mathrm{m}$ PDMS/DVB SPME fiber (Supelco) was conditioned at $250^{\circ} \mathrm{C}$ overnight. Sampling was performed at $30^{\circ} \mathrm{C}$ by placing the fiber through the Mininert Valve into the headspace above the fungal culture for $3 \mathrm{~min}$. Vials were continuously swirled at $100 \mathrm{rpm}$ during incubation and SPME exposure. GC/MS parameters. Volatiles were desorbed from the fiber in a gas chromatograph (HP-6890, Hewlett-Packard Co., Wilmington, DE) injection port for 3 min; absorption and desorption time was optimized as described in [42]. Volatiles were separated on a $29 \mathrm{~m} /$ $250 \mu \mathrm{m}$ i.d. capillary column HP-5MS (Hewlett-Packard Co., Wilmington, DE) having a film thickness $0.25 \mu \mathrm{m}$. The first $20 \mathrm{~cm}$ of the column was cooled with liquid nitrogen during the desorption process to cryofocus the volatiles. Ultrapure helium (99.999\%) was used as a carrier gas at a flow rate of $1.5 \mathrm{ml} / \mathrm{min}$. The initial temperature of the column $\left(40^{\circ} \mathrm{C}\right)$ was increased upon removal of liquid nitrogen at $60^{\circ} \mathrm{C} / \mathrm{min}$ to obtain a final temperature of $250^{\circ} \mathrm{C}$, which was maintained for $1 \mathrm{~min}$. Following chromatographic separation, metabolites were fragmented with an electron ionization source and ion masses were detected by time-of-flight mass spectrometry (FCD-650, LECO Corp., St. Joseph, MI). Preliminary identification of metabolites was achieved by comparison of their mass spectra with those of authenticated chemical standards contained in a mass spectrum library (National Institute for Standard Technology, Search 
Version 1.5, Gaithersburg, MD). A total of 4 to10 biological replicates were performed for each strain and condition. Only compounds detected in $50 \%$ or more replicates were then confirmed by comparison of their GC retention time, MS ion spectra and retention index (RI). Finally, the compounds with probability values below $70 \%$ were rejected.

\section{Ethanol measurements}

Twelve $\mathrm{ml}$ of fungal culture were dispensed into $22 \mathrm{ml}$ clear screw cap vials equipped with Mininert ${ }^{\circ}$ Valves (all from Supelco). Vials were incubated at $30^{\circ} \mathrm{C}$ for at least $30 \mathrm{~min}$ before headspace gases were sampled and ethanol levels were determined by means of gas chromatography (GC) using ethanol standards as described previously [80].

\section{Assessment of sclerotia production}

Small $(60 \times 15 \mathrm{~mm})$ agar plates were center-inoculated with $10^{4}$ conidiospores and placed into a large Petri dish $(150 \times 15 \mathrm{~mm})$ as described above. The cultures were incubated at $30^{\circ} \mathrm{C}$ in the dark at $90 \%$ relative humidity. After 9 to 17 days, the colonies were sprayed with $95 \%$ ethanol to enhance visualization of sclerotia. The number of sclerotia per plate was assessed. The viability of sclerotia was tested by placing 5 randomly chosen sclerotia onto YES agar medium which was incubated for 7 days in the dark.

\section{Feeding of branched chain amino acids}

Conidiospores $\left(10^{4} / \mathrm{ml}\right)$ were inoculated into YES liquid medium and incubated for $48 \mathrm{~h}$ at $30^{\circ} \mathrm{C}$ as described above. Sterile solutions of L-leucine, L-isoleucine, or Lvaline (all from Sigma, St. Louis, MO) in YES liquid medium were added to a final concentration of $0.03 \mathrm{M}$ and incubation continued for an additional $18 \mathrm{~h}$. Analysis of volatiles was conducted as described above.

\section{Analysis of leucine, isoleucine, and valine accumulation using LC/MS/MS}

Cultures were inoculated into YES liquid medium and incubated for designated periods of time at $30^{\circ} \mathrm{C}$ as described above. $1 \mathrm{ml}$ of each culture (containing medium plus mycelia) was extracted with $10 \mathrm{ml}$ solvent (acetonitril:isopropanol:water $=3: 3: 2$ ) for $1 \mathrm{~h}$ at RT. The extract was filtered through Whatman \#1 filter paper, then through a $0.45 \mu \mathrm{m}$ sterile filter (MILLEX ${ }^{\odot} \mathrm{HA}$, Millipore, Carrigtwohill, Co. Cork, Ireland); the extract was stored at $-20^{\circ} \mathrm{C}$. Ten $\mu$ of each extract were analyzed by a 3200 Q-Trap LC/MS/MS system (Applied Biosystems, Foster City, CA) at the RTSF/Mass Spectrometry Facility, MSU, using a ZIC-pHILIC column (SeQuant Merck, Darmstadt, Germany); acetonitril and $10 \mathrm{mM}$ ammonium acetate in $\mathrm{H}_{2} \mathrm{O}$ were used as solvents with gradients of acetonitrile $98 \%, 50 \%, 5 \%$.

\section{Analysis of gene expression using RT-PCR}

Total RNA extraction and preparation of cDNA was conducted as described elsewhere [14]. Primers (Figure 6) were designed based on an A. flavus genome database http:// www.aspergillusflavus.org; the $A$. flavus genome exhibits 95-98\% similarity to the $A$. parasiticus genome [12,14].

\section{Additional material}

Additional file 1: Figure S1 - Growth of $A$. parasiticus strains in YES liquid medium. Conidiospores were inoculated into $100 \mathrm{ml}$ of liquid YES medium at $10^{4} / \mathrm{ml}$ and the cultures were grown at $30^{\circ} \mathrm{C}$, with shaking at150 rpm, in the dark for designated periods of time. Dry weight was estimated as described in Methods.

Additional file 2: Figure S2 - SPME-GC/MS headspace gas analysis of selected volatile compounds produced by aspergilli grown in YES medium in the dark for $\mathbf{7 2} \mathbf{~ h}$. Conidiospores were inoculated into $100 \mathrm{ml}$ of liquid YES medium at $10^{4} / \mathrm{ml}$ and the cultures were grown at $30^{\circ} \mathrm{C}$, with shaking at $150 \mathrm{rpm}$, in the dark for $72 \mathrm{~h}$. Each culture was grown in two individual flasks. Each experiment was conducted in triplicate. The results are presented as an average of six measurements of relative peak area $\times 10^{4}+$ S.E. R.T., retention time, sec.

Additional file 3: Figure S3 - Production of fungal volatiles through pathways of branched chain amino acid catabolism. 2-ketoacids, the main intermediates, are formed through enzymatic transamination of branched chain amino acids; they can also be synthesized de novo. 2keto acid decarboxylase leads to formation of the corresponding alcohols. 2-ketoacid dehydrogenase leads to formation of the corresponding CoA derivatives and, subsequently to methyl and ethyl esters.

Additional file 4: Figure S4 - Branched chain amino acid-derived volatiles generated by SU-1 grown for $48 \mathrm{~h}$ and $\mathbf{7 2} \mathrm{h}$ in light. Conidiospores were inoculated into $100 \mathrm{ml}$ of liquid YES medium at $10^{4}$ / $\mathrm{ml}$ and the cultures were grown at $30^{\circ} \mathrm{C}$, with shaking at150 rpm, in the light for $48 \mathrm{~h}$ and $72 \mathrm{~h}$. Volatiles were analyzed as described in Methods.

Additional file 5: Figure S5 - Branched chain amino acid-derived esters detected in $\boldsymbol{A}$. parasiticus strains. ${ }^{*}$, esters unique to $\triangle v e A$.

Additional file 6: Figure S6 - Amino acid sequence alignment of the putative $A$. flavus branched chain amino acid aminotransferases AFLA_113800 and AFLA_044190 with yeast BAT1 and BAT2. Amino acid sequences were aligned using Clustal multiple sequence alignment program. AFLA_113800 exhibits 61\% identity to BAT1 and 60\% identity to BAT2. AFLA_044190 exhibits $43 \%$ identity to BAT1 and $44 \%$ identity to BAT2. The highlighted lysine residue represents the active site of the protein in E.coli. An asterisk was added below the sequences at conserved amino acid.

Additional file 7: Figure S7 - Amino acid sequence alignment of the putative A. flavus alcohol dehydrogenase, AFLA_048690, with the yeast alcohol dehydrogenase, ADH1. Amino acid sequences were aligned using Clustal multiple sequence alignment program.

AFLA_048690 exhibits 57\% identity to yeast ADH1. An asterisk was added below the sequences at conserved amino acid.

\section{Abbreviations}

SPME-GC/MS: solid phase microextraction - gas chromatography/mass spectrometry; NIST: National Institute for Standard Technology; YES: yeast extract sucrose; GMS: glucose minimal salts.

\section{Acknowledgements}

We thank Dr. Ana M. Calvo (Department of Biological Sciences, Northern Illinois University, DeKalb, IL) for providing the $\Delta v e A$ strain. We thank the reviewers for their input, which improved our work significantly. This work was supported by funds from the Michigan Agricultural Experiment Station. 


\section{Author details}

'Department of Food Science and Human Nutrition, Michigan State University, East Lansing, MI, USA. 'Department of Microbiology and Molecular Genetics, Michigan State University, East Lansing, MI, USA. ${ }^{3}$ Department of Horticulture, Michigan State University, East Lansing, MI, USA. ${ }^{4}$ Department of Biochemistry and Molecular Biology, Michigan State University, East Lansing, MI, USA. ${ }^{5}$ Department of Chemistry, Michigan State University, East Lansing, MI, USA. ${ }^{6}$ National Food Safety and Toxicology Center, Michigan State University, East Lansing, MI, USA.

\section{Authors' contributions}

LVR and JEL designed research; LVR, AC, AAK, AVK, DA, and DV performed research; $\mathrm{ML}, \mathrm{RMB}$, and $\mathrm{ADJ}$ contributed new technology/analytic tools; LVR and AVK analyzed the data; LVR and JEL wrote the paper. All authors read and approved the final manuscript.

Received: 2 June 2010 Accepted: 24 August 2010

Published: 24 August 2010

\section{References}

1. Frisvad JC, Andersen B, Thrane U: The use of secondary metabolite profiling in chemotaxonomy of filamentous fungi. Mycol Res 2008, 112(Pt 2):231-240.

2. Frisvad JC, Larsen TO, de Vries R, Meijer M, Houbraken J, Cabanes FJ, Ehrlich K, Samson RA: Secondary metabolite profiling, growth profiles and other tools for species recognition and important Aspergillus mycotoxins. Stud Mycol 2007, 59:31-37.

3. Thrane U, Andersen B, Frisvad JC, Smedsgaard J: The exo-metabolome in filamentous fungi. In Metabolomics. Edited by: Nielsen J. AJMC, Berlin Heidelberg: Springer-Verlag; 2007:18:235-252.

4. Roze LV, Chanda A, Linz JE: Compartmentalization and molecular traffic in secondary metabolism: A new understanding of established cellular processes. Fungal Genet Biol 2010.

5. Brakhage AA, Schroeckh V: Fungal secondary metabolites - Strategies to activate silent gene clusters. Fungal Genet Biol 2010

6. Firn RD, Jones CG: A Darwinian view of metabolism: molecular properties determine fitness. J Exp Bot 2009, 60(3):719-726.

7. Kroken S: Evolution of Secondary Metabolism in Microbes. Annu Rev Phytopathol 2009.

8. Price-Whelan A, Dietrich LE, Newman DK: Rethinking 'secondary' metabolism: physiological roles for phenazine antibiotics. Nat Chem Biol 2006, 2(2):71-78

9. Schroeckh V, Scherlach K, Nutzmann HW, Shelest E, Schmidt-Heck W, Schuemann J, Martin K, Hertweck C, Brakhage AA: Intimate bacterial-fungal interaction triggers biosynthesis of archetypal polyketides in Aspergillus nidulans. Proc Natl Acad Sci USA 2009, 106(34):14558-14563.

10. Vining LC: Secondary metabolism, inventive evolution and biochemical diversity-a review. Gene 1992, 115(1-2):135-140.

11. Bok JW, Chiang YM, Szewczyk E, Reyes-Dominguez Y, Davidson AD, Sanchez JF, Lo HC, Watanabe K, Strauss J, Oakley BR, et al: Chromatin-level regulation of biosynthetic gene clusters. Nat Chem Biol 2009, 5(7):462-464.

12. Chanda A, Roze LV, Kang S, Artymovich KA, Hicks GR, Raikhel NV, Calvo AM, Linz JE: A key role for vesicles in fungal secondary metabolism. Proc Natl Acad Sci USA 2009, 106(46):19533-19538.

13. Georgianna DR, Payne GA: Genetic regulation of aflatoxin biosynthesis: from gene to genome. Fungal Genet Biol 2009, 46(2):113-125.

14. Roze LV, Arthur AE, Hong SY, Chanda A, Linz JE: The initiation and pattern of spread of histone $\mathrm{H} 4$ acetylation parallel the order of transcriptional activation of genes in the aflatoxin cluster. Mol Microbiol 2007, 66(3):713-726.

15. Roze LV, Miller MJ, Rarick M, Mahanti N, Linz JE: A novel CAMP-response element, CRE1, modulates expression of nor-1 in Aspergillus parasiticus. J Biol Chem 2004, 279(26):27428-27439.

16. Duran RM, Cary JW, Calvo AM: Production of cyclopiazonic acid, aflatrem, and aflatoxin by Aspergillus flavus is regulated by veA, a gene necessary for sclerotial formation. Appl Microbiol Biotechnol 2007, 73(5):1158-1168.

17. Tokuoka M, Seshime Y, Fujii I, Kitamoto K, Takahashi T, Koyama Y: Identification of a novel polyketide synthase-nonribosomal peptide synthetase (PKS-NRPS) gene required for the biosynthesis of cyclopiazonic acid in Aspergillus oryzae. Fungal Genet Biol 2008, 45(12):1608-1615.
18. Chang PK, Horn BW, Dorner JW: Clustered genes involved in cyclopiazonic acid production are next to the aflatoxin biosynthesis gene cluster in Aspergillus flavus. Fungal Genet Biol 2009, 46(2):176-182.

19. Roze LV, Beaudry RM, Keller NP, Linz JE: Regulation of aflatoxin synthesis by FadA/cAMP/protein kinase A signaling in Aspergillus parasiticus. Mycopathologia 2004, 158(2):219-232.

20. Calvo AM, Wilson RA, Bok JW, Keller NP: Relationship between secondary metabolism and fungal development. Microbiol Mol Biol Rev 2002, 66(3):447-459.

21. Kale SP, Milde L, Trapp MK, Frisvad JC, Keller NP, Bok JW: Requirement of LaeA for secondary metabolism and sclerotial production in Aspergillus flavus. Fungal Genet Biol 2008, 45(10):1422-1429.

22. Mahanti N, Bhatnagar D, Cary JW, Joubran J, Linz JE: Structure and function of fas-1A, a gene encoding a putative fatty acid synthetase directly involved in aflatoxin biosynthesis in Aspergillus parasiticus. Appl Environ Microbiol 1996, 62(1):191-195.

23. Woloshuk CP, Foutz KR, Brewer JF, Bhatnagar D, Cleveland TE, Payne GA: Molecular characterization of aflR, a regulatory locus for aflatoxin biosynthesis. Appl Environ Microbiol 1994, 60(7):2408-2414.

24. Bayram O, Krappmann S, Ni M, Bok JW, Helmstaedt K, Valerius O, BrausStromeyer S, Kwon NJ, Keller NP, Yu JH, et al: VelB/VeA/LaeA complex coordinates light signal with fungal development and secondary metabolism. Science 2008, 320(5882):1504-1506.

25. Maggio-Hall LA, Wilson RA, Keller NP: Fundamental contribution of betaoxidation to polyketide mycotoxin production in planta. Mol Plant Microbe Interact 2005, 18(8):783-793.

26. Ehrlich KC: Evolution of the aflatoxin gene cluster. Mycotoxin Research 2006, 22(1):9-15

27. Huang JQ, Jiang HF, Zhou YQ, Lei Y, Wang SY, Liao BS: Ethylene inhibited aflatoxin biosynthesis is due to oxidative stress alleviation and related to glutathione redox state changes in Aspergillus flavus. Int J Food Microbiol 2009, 130(1):17-21

28. Kim JH, Yu J, Mahoney N, Chan KL, Molyneux RJ, Varga J, Bhatnagar D, Cleveland TE, Nierman WC, Campbell BC: Elucidation of the functional genomics of antioxidant-based inhibition of aflatoxin biosynthesis. Int $\mathrm{J}$ Food Microbiol 2008, 122(1-2):49-60

29. Narasaiah KV, Sashidhar RB, Subramanyam C: Biochemical analysis of oxidative stress in the production of aflatoxin and its precursor intermediates. Mycopathologia 2006, 162(3):179-189.

30. Rohlfs M, Albert M, Keller NP, Kempken F: Secondary chemicals protect mould from fungivory. Biol Lett 2007, 3(5):523-525.

31. Bhatnagar D, Ehrlich KC, Cleveland TE: Molecular genetic analysis and regulation of aflatoxin biosynthesis. Appl Microbiol Biotechnol 2003, 61(2):83-93.

32. Calvo AM, Bok J, Brooks W, Keller NP: veA is required for toxin and sclerotial production in Aspergillus parasiticus. Appl Environ Microbiol 2004, 70(8):4733-4739

33. Cary JW, Dyer JM, Ehrlich KC, Wright MS, Liang SH, Linz JE: Molecular and functional characterization of a second copy of the aflatoxin regulatory gene, aflR-2, from Aspergillus parasiticus. Biochim Biophys Acta 2002, 1576(3):316-323

34. Trail F, Mahanti N, Linz J: Molecular biology of aflatoxin biosynthesis. Microbiology 1995, 141(Pt 4):755-765.

35. Calvo AM, Gardner HW, Keller NP: Genetic connection between fatty acid metabolism and sporulation in Aspergillus nidulans. J Biol Chem 2001, 276(28):25766-25774

36. Cary JW, Ehrlich KC: Aflatoxigenicity in Aspergillus: molecular genetics, phylogenetic relationships and evolutionary implications. Mycopathologia 2006, 162(3):167-177.

37. Cary JW, Ehrlich KC, Bland JM, Montalbano BG: The aflatoxin biosynthesis cluster gene, afIX, encodes an oxidoreductase involved in conversion of versicolorin A to demethylsterigmatocystin. Appl Environ Microbiol 2006, 72(2):1096-1101.

38. Maggio-Hall LA, Keller NP: Mitochondrial beta-oxidation in Aspergillus nidulans. Mol Microbiol 2004, 54(5):1173-1185.

39. Maggio-Hall LA, Lyne P, Wolff JA, Keller NP: A single acyl-CoA dehydrogenase is required for catabolism of isoleucine, valine and short-chain fatty acids in Aspergillus nidulans. Fungal Genet Biol 2008, 45(3):180-189

40. Last RL, Jones AD, Shachar-Hill Y: Towards the plant metabolome and beyond. Nat Rev Mol Cell Biol 2007, 8(2):167-174. 
41. Goodridge CF, Beaudry RM, Pestka JJ, Smith DM: Solid phase microextraction-gas chromatography for quantifying headspace hexanal above freeze-dried chicken myofibrils. J Agric Food Chem 2003, 51(15):4185-4190.

42. Song J, Fan LH, Beaudry RM: Application of solid phase microextraction and gas chromatography time-of-flight mass spectrometry for rapid analysis of flavor volatiles in tomato and strawberry fruits. Journal of Agricultural and Food Chemistry 1998, 46(9):3721-3726.

43. Tikunov $Y$, Lommen $A$, de $\operatorname{Vos} C H$, Verhoeven $H A$, Bino RJ, Hall RD, Bovy AG: A novel approach for nontargeted data analysis for metabolomics. Large-scale profiling of tomato fruit volatiles. Plant Physiol 2005, 139(3):1125-1137.

44. Qualley AV, Dudareva N: Metabolomics of plant volatiles. Methods Mol Biol 2009, 553:329-343.

45. Rossouw D, Naes T, Bauer FF: Linking gene regulation and the exometabolome: a comparative transcriptomics approach to identify genes that impact on the production of volatile aroma compounds in yeast. BMC Genomics 2008, 9:530.

46. Bino RJ, Hall RD, Fiehn O, Kopka J, Saito K, Draper J, Nikolau BJ, Mendes P, Roessner-Tunali $U$, Beale $\mathrm{MH}$, et al: Potential of metabolomics as a functional genomics tool. Trends Plant Sci 2004, 9(9):418-425.

47. Dudareva N, Pichersky E: Metabolic engineering of plant volatiles. Curr Opin Biotechnol 2008, 19(2):181-189.

48. Fiehn O, Kopka J, Trethewey RN, Willmitzer L: Identification of uncommon plant metabolites based on calculation of elemental compositions using gas chromatography and quadrupole mass spectrometry. Anal Chem 2000, 72(15):3573-3580.

49. Berger RG: Flavours and fragrances: chemistry, bioprocessing and sustainability. Berlin Heidelberg New-York: Springer 2007.

50. Kondo T, Sakurada M, Okamoto S, Ono M, Tsukigi $H$, Suzuki A, Nagasawa $H$, Sakuda S: Effects of aflastatin A, an inhibitor of aflatoxin production, on aflatoxin biosynthetic pathway and glucose metabolism in Aspergillus parasiticus. J Antibiot (Tokyo) 2001, 54(8):650-657.

51. Ono M, Sakuda S, Suzuki A, Isogai A: Aflastatin A, a novel inhibitor of aflatoxin production by aflatoxigenic fungi. J Antibiot (Tokyo) 1997, 50(2):111-118

52. Calvo AM: The VeA regulatory system and its role in morphological and chemical development in fungi. Fungal Genet Biol 2008, 45(7):1053-1061.

53. Roze LV, Beaudry RM, Arthur AE, Calvo AM, Linz JE: Aspergillus volatiles regulate aflatoxin synthesis and asexual sporulation in Aspergillus parasiticus. Appl Environ Microbiol 2007, 73(22):7268-7276.

54. Hazelwood LA, Daran JM, van Maris AJ, Pronk JT, Dickinson JR: The Ehrlich pathway for fusel alcohol production: a century of research on Saccharomyces cerevisiae metabolism. Appl Environ Microbiol 2008, 74(8):2259-2266.

55. Leskovac $V$, Trivic S, Pericin D: The three zinc-containing alcohol dehydrogenases from baker's yeast, Saccharomyces cerevisiae. FEMS Yeast Res 2002, 2(4):481-494.

56. Dickinson JR, Salgado LE, Hewlins MJ: The catabolism of amino acids to long chain and complex alcohols in Saccharomyces cerevisiae. J Biol Chem 2003, 278(10):8028-8034.

57. Zhang $Y Q$, Brock M, Keller NP: Connection of propionyl-CoA metabolism to polyketide biosynthesis in Aspergillus nidulans. Genetics 2004 168(2):785-794.

58. Zhang YQ, Keller NP: Blockage of methylcitrate cycle inhibits polyketide production in Aspergillus nidulans. Mol Microbiol 2004, 52(2):541-550.

59. Amaike S, Keller NP: Distinct roles for VeA and LaeA in development and pathogenesis of Aspergillus flavus. Eukaryot Cell 2009, 8(7):1051-1060.

60. Brock M, Fischer R, Linder D, Buckel W: Methylcitrate synthase from Aspergillus nidulans: implications for propionate as an antifungal agent. Mol Microbiol 2000, 35(5):961-973.

61. Gerike U, Hough DW, Russell NJ, Dyall-Smith ML, Danson MJ: Citrate synthase and 2-methylcitrate synthase: structural, functional and evolutionary relationships. Microbiology 1998, 144(Pt 4):929-935.

62. Maerker C, Rohde M, Brakhage AA, Brock M: Methylcitrate synthase from Aspergillus fumigatus. Propionyl-CoA affects polyketide synthesis, growth and morphology of conidia. Febs J 2005, 272(14):3615-3630.

63. Pronk JT, van der Linden-Beuman A, Verduyn C, Scheffers WA, van Dijken JP: Propionate metabolism in Saccharomyces cerevisiae: implications for the metabolon hypothesis. Microbiology 1994, 140(Pt 4):717-722.
64. Keurentjes JJ: Genetical metabolomics: closing in on phenotypes. Curr Opin Plant Biol 2009, 12(2):223-230.

65. Mo ML, Palsson BO, Herrgard MJ: Connecting extracellular metabolomic measurements to intracellular flux states in yeast. BMC Syst Biol 2009, 3:37.

66. Weckwerth $\mathrm{W}$ : Integration of metabolomics and proteomics in molecular plant physiology-coping with the complexity by data-dimensionality reduction. Physiol Plant 2008, 132(2):176-189.

67. Andersen MR, Nielsen J: Current status of systems biology in aspergilli. Fungal Genet Biol 2009, 46(Suppl 1):S180-190.

68. Bhatanagar D, Rajasekaran K, Payne GA, Brown R, Yu J, Cleveland T: The "omics" Tools: Genomics, Proteomics, Metabolomics for Solving the Aflatoxin Contamination Problem. World Mycotoxin Journal 2008, 1(1):3-12.

69. Smedsgaard J, Nielsen J: Metabolite profiling of fungi and yeast: from phenotype to metabolome by MS and informatics. J Exp Bot 2005, 56(410):273-286

70. Panagiotou G, Villas-Boas SG, Christakopoulos P, Nielsen J, Olsson L: Intracellular metabolite profiling of Fusarium oxysporum converting glucose to ethanol. J Biotechnol 2005, 115(4):425-434.

71. Denoya CD, Fedechko RW, Hafner EW, McArthur HA, Morgenstern MR, Skinner DD, Stutzman-Engwall K, Wax RG, Wernau WC: A second branched-chain alpha-keto acid dehydrogenase gene cluster (bkdFGH) from Streptomyces avermitilis: its relationship to avermectin biosynthesis and the construction of a bkdF mutant suitable for the production of novel antiparasitic avermectins. J Bacteriol 1995, 177(12):3504-3511.

72. Stirrett K, Denoya C, Westpheling J: Branched-chain amino acid catabolism provides precursors for the Type II polyketide antibiotic, actinorhodin, via pathways that are nutrient dependent. J Ind Microbiol Biotechnol 2009, 36(1):129-137.

73. Ryan ED, Kohlhaw GB: Subcellular localization of isoleucine-valine biosynthetic enzymes in yeast. J Bacteriol 1974, 120(2):631-637.

74. Cary JW, GR OB, Nielsen DM, Nierman W, Harris-Coward P, Yu J, Bhatnagar D, Cleveland TE, Payne GA, Calvo AM: Elucidation of veAdependent genes associated with aflatoxin and sclerotial production in Aspergillus flavus by functional genomics. Appl Microbiol Biotechnol 2007, 76(5):1107-1118

75. Cary JW, Ehrlich KC, Wright M, Chang PK, Bhatnagar D: Generation of afIR disruption mutants of Aspergillus parasiticus. Appl Microbiol Biotechnol 2000, 53(6):680-684

76. Bennett JW, Fernholz FA, Lee LS: Effect of light on aflatoxins, anthraquinones, and sclerotia in Aspergillus flavus and A. parasiticus. Mycologia 1978, 70(1):104-116.

77. Hong SY, Linz JE: Functional expression and subcellular localization of the aflatoxin pathway enzyme Ver-1 fused to enhanced green fluorescent protein. Appl Environ Microbiol 2008, 74(20):6385-6396.

78. Skory CD, Chang PK, Cary J, Linz JE: Isolation and characterization of a gene from Aspergillus parasiticus associated with the conversion of versicolorin A to sterigmatocystin in aflatoxin biosynthesis. Appl Environ Microbiol 1992, 58(11):3527-3537.

79. Buchanan RL, Lewis DF: Regulation of aflatoxin biosynthesis: effect of glucose on activities of various glycolytic enzymes. Appl Environ Microbiol 1984, 48(2):306-310.

80. Roze LV, Calvo AM, Gunterus A, Beaudry R, Kall M, Linz JE: Ethylene modulates development and toxin biosynthesis in Aspergillus possibly via an ethylene sensor-mediated signaling pathway. J Food Prot 2004, 67(3):438-447.

81. Butchko RA, Adams TH, Keller NP: Aspergillus nidulans mutants defective in stc gene cluster regulation. Genetics 1999, 153(2):715-720.

doi:10.1186/1471-2091-11-33

Cite this article as: Roze et al: Volatile profiling reveals intracellular metabolic changes in Aspergillus parasiticus: veA regulates branched chain amino acid and ethanol metabolism. BMC Biochemistry 2010 11:33. 